\title{
Immunotherapy Response Assessment in Neuro-Oncology (iRANO): A Report of the RANO Working Group
}

\author{
Hideho Okada, MD ${ }^{1}$, Michael Weller, MD², Raymond Huang, MD $^{3}$, Gaetano Finocchiaro, \\ $\mathrm{MD}^{4}$, Mark R. Gilbert, $\mathrm{MD}^{5}$, Wolfgang Wick, $\mathrm{MD}^{6}$, Benjamin M. Ellingson, $\mathrm{PhD}^{7}$, Naoya \\ Hashimoto, $\mathbf{M D}^{8}$, Ian F. Pollack, $\mathbf{M D}^{9}$, Alba A. Brandes, $\mathbf{M D}^{10}$, Enrico Franceschi, $\mathbf{M D}^{10}$, \\ Christel Herold-Mende, PhD ${ }^{11}$, Lakshmi Nayak, MD ${ }^{12}$, Ashok Panigrahy, MD ${ }^{13}$, Whitney B. \\ Pope, MD ${ }^{14}$, Robert Prins, PhD ${ }^{15}$, John H. Sampson, MD ${ }^{16}$, Patrick Y. Wen, MD ${ }^{12}$, and David \\ A. Reardon, MD $^{12}$
}

${ }^{1}$ Department of Neurological Surgery, University of California, San Francisco, San Francisco, CA, USA ${ }^{2}$ Department of Neurology, University Hospital Zurich, Zurich, Switzerland ${ }^{3}$ Department of Radiology, Brigham and Women's Hospital, Boston, MA, USA ${ }^{4}$ Department of Neuro-Oncology, Istituto Neurologico Besta, Milan, Italy ${ }^{5}$ Neuro-Oncology Branch, National Institutes of Health, Bethesda, MD, USA ${ }^{6}$ Department of Neurooncology, Heidelberg University Hospital, Heidelberg, Germany ${ }^{7}$ Departments of Radiological Sciences, Bioengineering, Biomedical Physics, and Psychiatry David Geffen School of Medicine University of California, Los Angeles Los Angeles, CA, USA ${ }^{8}$ Department of Neurosurgery, Osaka University Graduate School of Medicine, Suita, Osaka, Japan ${ }^{9}$ Department of Neurological Surgery, University of Pittsburgh School of Medicine, Children's Hospital of Pittsburgh, Pittsburgh, PA, USA ${ }^{10}$ Department of Medical Oncology, Azienda USL-IRCCS Institute of Neurological Science, Bologna, Italy ${ }^{11}$ Department of Neurosurgery, Division of Experimental Neurosurgery, University of Heidelberg, Heidelberg, Germany ${ }^{12}$ Center for Neuro-Oncology, Dana-Farber Cancer Institute, Boston, MA, USA ${ }^{13}$ Department of Radiology, University of Pittsburgh School of Medicine, Children's Hospital of Pittsburgh, Pittsburgh, PA, USA ${ }^{14}$ Department of Radiology, David Geffen School of Medicine at University of California, Los Angeles, Los Angeles, CA, USA ${ }^{15}$ Department of Neurosurgery, David Geffen School of Medicine at University of California, Los Angeles, Los Angeles, CA, USA ${ }^{16}$ Department of Neurosurgery, Duke University School of Medicine, Durham, NC, USA

\footnotetext{
This manuscript version is made available under the CC BY-NC-ND 4.0 license.

Corresponding Authors: Hideho Okada, MD, PhD, University of California, San Francisco, Department of Neurological Surgery, $14503^{\text {rd }}$ Street, San Francisco, CA 94158-0520, Telephone: 415-476-1637, hideho.okada@ ucsf.edu; David A. Reardon, MD, Center for Neuro-Oncology, Dana-Farber Cancer Institute, 450 Brookline Avenue, D2134, Boston, MA 02215, Telephone: 617-632-2166, Facsimile: 617-632-4773, david_reardon@dfci.harvard.edu.

Publisher's Disclaimer: This is a PDF file of an unedited manuscript that has been accepted for publication. As a service to our customers we are providing this early version of the manuscript. The manuscript will undergo copyediting, typesetting, and review of the resulting proof before it is published in its final citable form. Please note that during the production process errors may be discovered which could affect the content, and all legal disclaimers that apply to the journal pertain.

Author Contributions All authors contributed to the conception and design of the guideline, writing, review and revision of the manuscript.

Declaration of interests The other authors declared no conflicts of interest.

The authors confirm that the paper has not been submitted to another journal, and has not been published in whole or in part elsewhere previously.
} 


\section{Abstract}

Immunotherapy represents a promising area of therapy among neuro-oncology patients. However, early phase studies reveal unique challenges associated with assessment of radiological changes reflecting delayed responses or therapy-induced inflammation. Clinical benefit, including longterm survival and tumor regression, can still occur following initial apparent progression or appearance of new lesions. Refinement of response assessment criteria for neuro-oncology patients undergoing immunotherapy is therefore warranted. A multinational and multidisciplinary panel of neuro-oncology immunotherapy experts describes immunotherapy response assessment for neuro-oncology (iRANO) criteria that are based on guidance for determination of tumor progression outlined by the immune-related response criteria (irRC) and the response assessment in neuro-oncology (RANO) working group. Among patients who demonstrate imaging findings meeting RANO criteria for progressive disease (PD) within six months of initiating immunotherapy including the development of new lesions, confirmation of radiographic progression on follow-up imaging is recommended provided that the patient is not significantly worse clinically. The proposed criteria also include guidelines for use of corticosteroids. The role of advanced imaging techniques and measurement of clinical benefit endpoints including neurologic and immunologic functions are reviewed. The iRANO guidelines put forth herein will evolve successively to improve their utility as further experience from immunotherapy trials in neuro-oncology accumulate.

\section{Keywords}

immunotherapy; vaccine; immune checkpoint; magnetic resonance imaging; pseudoprogression

\section{Introduction}

Immunotherapy for cancer has made exciting recent progress. The United States Food and Drug Administration (FDA) approved the first vaccine against non-viral cancers (sipuleucel$\mathrm{T})^{1}$ and blocking monoclonal antibodies $(\mathrm{mAb})$ to the immune checkpoint molecules cytotoxic T lymphocyte-4 (CTLA-4; ipilimumab) and programmed death 1 (PD-1; pembroluzimab and nivolumab) for metastatic melanoma as well as non-small cell lung cancer (NSCLC). ${ }^{2-5}$ Chimeric antigen receptor (CAR) engineered autologous $\mathrm{T}$ cells have induced durable remissions among leukemia patients refractory to conventional therapies including bone marrow transplantation. ${ }^{6,7}$ For patients with primary and metastatic neurooncology malignancies, clinical trials evaluating a variety of immunotherapeutic approaches are underway, and promising preliminary results are emerging. ${ }^{8-10}$

\section{Ongoing Evolution of Response Assessment in Neuro-Oncology}

Traditional imaging response assessment tools, including World Health Organization $(\mathrm{WHO})^{11}$, Response Evaluation in Solid Tumors (RECIST) ${ }^{12}$, and Macdonald criteria ${ }^{13}$, originated in the cytotoxic therapy era where radiographic findings directly reflected antitumor effect. As oncology treatments have expanded beyond cytotoxic therapy, the impact of therapeutics on tumor imaging findings has become less straightforward. For neurooncology, pseudoprogression following radiotherapy and temozolomide chemotherapy 
$(\mathrm{TMZ} / \mathrm{RT} \rightarrow \mathrm{TMZ})^{14}$, and pseudoresponse following anti-angiogenic agents ${ }^{15}$, highlight challenges with interpreting imaging changes in the modern era. The response assessment in neuro-oncology (RANO) criteria ${ }^{16}$ were proposed in 2010 to better assess evolving complexities of imaging assessment for malignant glioma patients. Subsequently, variations of RANO were refined for patients with low-grade glioma ${ }^{17}$ and brain metastases. ${ }^{18}$

A key cornerstone of RANO is guidance for the phenomenon of pseudoprogression which occurs in approximately 10-20\% of newly diagnosed glioblastoma patients following $\mathrm{TMZ} / \mathrm{RT} \rightarrow \mathrm{TMZ}^{14,19-21}$ The precise mechanism of pseudoprogression remains poorly understood, but most cases peak within three months of completing chemoradiation, although longer time courses have been reported. ${ }^{19}$ Thereafter, radiographic changes may stabilize and ultimately improve. RANO guidelines have been widely adopted in daily practice and clinical research. Specifically, RANO defines that progressive disease (PD) should be diagnosed radiographically no sooner than three months following completion of concomitant TMZ/RT, unless there is: 1) new enhancement outside the main radiation field or; 2) pathologic confirmation of unequivocal tumor progression. Furthermore, RANO permits patients with progressive radiographic findings of unclear etiology to continue current therapy pending follow-up imaging.

Important issues regarding progressive imaging findings among neuro-oncology patients treated with immunotherapy suggest that further adaptation of RANO is warranted. First, the mechanism underlying pseudoprogression following immunotherapy is likely distinct from that associated with TMZ/RT $\rightarrow$ TMZ, with important differences in kinetics, frequency, and overall impact for patients. For example, although the temporal window for TMZ/ $\mathrm{RT} \rightarrow \mathrm{TMZ}$ pseudoprogression generally peaks within three months, the time frame for immunotherapy-associated pseudoprogression remains to be defined and may differ by class of immunotherapeutic. Second, RANO does not permit treatment continuation beyond actual tumor progression because subsequent therapeutic benefit supporting this practice has not been documented for oncology treatments other than immunotherapies. Third, appearance of a new lesion outside the main radiation field automatically defines PD in the RANO criteria. Finally, RANO does not require a repeat scan to confirm PD.

\section{Challenges Interpreting Worsened Radiographic Findings Following Immunotherapy}

The interpretation of decreased size of an enhancing lesion is straightforward as such changes indicate a true anti-tumor effect because immunotherapeutics are not associated with pseudoresponse. In contrast, correct interpretation of progressive imaging findings following administration of immunotherapeutics is essential since early progressive radiographic changes do not always preclude subsequent therapeutic benefit. ${ }^{22-32}$ There are two main explanations for a possible disconnect between worsened early imaging findings and subsequent therapeutic benefit. First, effective immune responses may require time to evolve, and early imaging may show true PD, including the development of new lesions. Nonetheless, once induced, an effective anti-tumor immune response may subsequently lead to clinical benefit. Second, because the mode of action may involve an inflammatory response in areas of macroscopic and microscopic infiltrative tumor, localized inflammatory 
responses can mimic radiologic features of tumor progression with increased enhancement and edema. ${ }^{33}$

In an evaluation of 487 advanced melanoma patients treated with ipilimumab in three phase II studies, four patterns of radiographic response were observed. ${ }^{22}$ Two of these response patterns were captured by conventional WHO or RECIST criteria including: a) radiologic response in baseline lesions with no new lesions; and b) stable disease, which was followed by slow progressive decrease in tumor burden among some patients. Two other previously unrecognized patterns of response were not captured by conventional response assessment criteria. In some patients, an increase in size of existing lesions was followed by radiographic response or stable disease without the addition of further therapy other than ipilimumab. Among other patients, new lesions were noted early on, but subsequent response or stable disease was later achieved without alternative therapeutic intervention. Additional recent examples also highlight the potential for early imaging worsening to be misleading in patients undergoing immunotherapy. First, spider plots evaluating percent change in target lesion size from baseline over time for individual patients treated with antiPD-1/PD-L1 therapy reveal enlargement of initial tumor or even new lesions in some melanoma patients prior to eventual decrease in tumor size. ${ }^{26,28,31}$ Second, in an evaluation of 227 patients treated with ipilimumab, 22 patients (9.7\%) who met WHO imaging criteria for PD subsequently demonstrated clinical benefit including five patients who ultimately achieved PR, and 17 with SD. ${ }^{22}$ In a phase II study of tremelimumab, another anti-CTLA-4 MAb, eight patients demonstrated a PR of target lesions by RECIST criteria concurrent with new lesions in six patients and progression of non-target lesions in two others. ${ }^{34}$ Of note, overall survival of these eight patients ranged from 21 to 39 months, whereas the median survival for all enrolled patients was 10.0 months. These examples underscore a potential disparity between early worsening on imaging assessment and ultimate clinical benefit including improved survival among patients treated with immunotherapy.

The frequency of ultimate clinical benefit following early progressive imaging findings among neuro-oncology patients undergoing immunotherapy approaches is unknown. Preliminary results of recently initiated clinical trials evaluating immune checkpoint blocking antibodies among recurrent glioblastoma patients and vaccines in WHO grade II low-grade glioma patients demonstrate that early progressive radiographic changes or appearance of new enhancing lesions may subsequently stabilize or disappear, respectively (Figure 1).

\section{New lesions}

Appearance of new lesions is a criterion that defines progression of disease by RANO as well as Macdonald criteria. However, transient appearance of new enhancing lesions at either local or distant sites may occur among neuro-oncologic patients receiving immunotherapies (Figure 1B). ${ }^{25,36}$ For cases of pseudoprogression, histopathology typically reveals remarkable immune cell infiltration, such as $\mathrm{CD} 8^{+} \mathrm{T}$ lymphocytes, but not mitotically active tumor cells. ${ }^{25}$ In such situations, careful radiologic and clinical assessments are warranted. In some cases, such new enhancing lesions may reflect immune responses directed against infiltrative brain tumor cells. 


\section{Confirmation of Radiographic Progression to Define Progressive Disease}

The immune-related response criteria (irRC) were issued to aid the interpretation of imaging changes among oncology patients undergoing immunotherapy. ${ }^{22,24,37}$ Their intent was to raise awareness that traditional imaging criteria to define PD may be less reliable and could lead to premature discontinuation of potentially beneficial therapy. A key component is the concept of confirmation of radiographic progression. irRC guidelines state that early increases in lesion size or new lesions do not define PD unless further progressive changes are confirmed upon follow-up imaging, provided that patients are not experiencing clinical decline. Confirmation to define PD is an important, novel aspect of irRC, although the converse, requirement of follow-up imaging to confirm a radiographic response, has been an accepted component of most response assessment metrics including RANO. Particularly for indications such as glioblastoma, where effective therapeutic interventions are limited and durable responses are elusive, continuation of immunotherapies beyond initial progression may lessen the likelihood of prematurely discontinuing potentially effective therapy. $2,22,24$

\section{When Is Confirmation of Radiographic Progression Appropriate?}

A critical issue is to identify patients who develop early progressive imaging findings, but still will derive therapeutic benefit from those truly resistant and unlikely to benefit. According to most response assessment criteria, including RANO, patients with significant neurologic decline, regardless of imaging findings, are deemed to have PD, providing their decline is not attributable to co-morbid events such as seizures or changes in medication, notably decreased corticosteroid dosing. For such patients, radiographic confirmation of PD is neither necessary nor appropriate and their date of PD is the date they developed significant neurologic decline attributable to underlying tumor. Future studies need to define the time window for patients without neurologic decline where early progressive imaging findings do not preclude subsequent clinical benefit. Experience among solid tumor patients treated with immune checkpoint blockade reveals that most patients who ultimately benefit demonstrate stable or improved radiographic findings within six months of initiating therapy, including those who exhibit early progressive radiographic findings. ${ }^{2,26,28,31}$ The kinetics of either pseudoprogression or delayed response following various types of immunotherapy among neuro-oncology patients require prospective evaluation. Nonetheless, anecdotal reports of glioma patients treated with tumor vaccination therapy have described pseudoprogressive radiographic findings that also typically manifest within six months of treatment initiation. ${ }^{25,36,38}$

Conversely, there is no evidence that patients develop delayed clinical benefit or radiographic response if they develop progressive radiographic findings more than six months after initiating immunotherapy. In order to determine whether a six month window to recommend confirmation of radiographic progression is appropriate, the iRANO working committee advocates that the timeframe of pseudoprogression be prospectively evaluated in future immunotherapy trials. 


\section{Three Months Period to Confirm Radiographic Progression}

Another critical unanswered question regarding the significance of early progressive imaging findings is how long such changes can evolve before clinicians can confidently conclude that they indicate PD. Is there a duration of time in which imaging findings may continue to worsen, but a given patient may still ultimately derive clinical benefit? Alternatively, how long should progressive imaging findings after initiation of immunotherapy be followed in order to confidently conclude that ultimate clinical benefit is unlikely?

The irRC guidelines recommend confirmation of progression with follow-up imaging at least four weeks from the initial scan documenting PD. ${ }^{22}$ Yet, four weeks may be too early to accurately ascertain the etiology of early progressive imaging changes and conclude that eventual clinical benefit is unlikely. In fact, spider-plots describing changes in tumor volume over time for solid tumor patients undergoing immune checkpoint blockade demonstrate that early progressive radiographic findings typically stabilize or improve within three months for the majority of patients who ultimately derive clinical benefit. ${ }^{26,28,31}$ Similarly, a three month window has been defined by RANO to determine the etiology of progressive imaging changes among malignant glioma patients following TMZ/RT $\rightarrow$ TMZ. ${ }^{14,39}$

Based on these observations, the iRANO working committee recommends that among patients with early progressive imaging findings including the development of new lesions who are not experiencing significant neurologic decline, confirmation of radiographic progression via follow-up imaging should be sought no sooner than three months after initial radiographic evidence of PD is detected, to decrease the likelihood of prematurely declaring PD in patients with pseudoprogression or delayed response. Imaging within the three months follow-up period can be performed as medically appropriate at the discretion of the treating clinician.

Among such patients, those with confirmation of further radiographic progression based on comparison to the scan which first revealed evidence of progression, or who exhibit significant clinical decline at any time, should be classified as progressive with the date of disease progression back-dated to the first date that the patient met criteria for radiographic progression. Such patients should be discontinued from their current immunotherapy regimen.

In the event that follow-up imaging does not confirm further progression compared to the scan which first revealed initial progressive changes, but instead reveals stabilization or reduction in tumor burden, in the absence of increased corticosteroid dosing, treatment should be continued or resumed.

Figure 2 provides an algorithm summarizing guidance on follow-up imaging following initial progressive changes. 


\section{Tissue Acquisition to Aid Response Assessment}

In uncertain cases in which acquisition of tumor histopathology via biopsy or resection is considered feasible, pathological assessment may be considered to clarify the etiology of progressive imaging findings. If pathology confirms a predominance of recurrent tumor, the etiology should be considered to be true progression. For cases where no evidence of viable tumor is detected, or where a prominence of gliosis/inflammation with limited viable tumor is observed, the etiology should be considered consistent with treatment effect, and such patients should be classified as stable and allowed to continue therapy.

Although considered a "gold standard", interpretation of tissue may be challenging. Biopsies typically acquire very small tissue aliquots and thus may be subject to "sampling artifact". In addition, many specimens will reveal mixed findings indicating the presence of viable tumor as well as treatment effect (inflammation, necrosis, etc.) and guidance on appropriate interpretation of such specimens is currently lacking. It will be critically important that neuropathologists and neuro-oncologists prospectively prioritize careful evaluation of histopathologic samples obtained from patients undergoing immunotherapy to better understand the significance of various patterns of mixed tissue findings.

\section{Immunotherapy Continuation Pending Confirmation of Progression}

Currently, it is not established whether continued immunotherapy following initial progression on imaging studies would provide treatment efficacy or harm to patients and further careful study of this important question is warranted. A decision whether a patient should continue immunotherapy pending confirmation of radiographic progression should be determined based on perceived benefits and risks. Continuation of immunotherapy may be considered pending follow-up imaging as long as patients are deriving apparent clinical benefit with minimal and acceptable toxicity. In contrast, clinicians may consider interrupting immunotherapy for patients who require a significant increase in corticosteroids (i.e. > $4 \mathrm{mg}$ of dexamethasone or equivalent per day) for evolving symptoms associated with cerebral edema or who are experiencing more than mild treatment-related toxicity such as immune related adverse events grade $\geq 2$.

Although somewhat arbitrarily set and not based on definitive data, these guidelines are included to limit the likelihood of progressive immunotherapy-induced inflammatory changes leading to significant deficits in otherwise stable or asymptomatic patients. In such patients, an interruption of immunotherapy dosing may be considered pending follow-up imaging. Further, one may opt to discontinue or interrupt immunotherapy at any time if this seems to be in the best medical interest of the patient. As general guidance, resumption of immunotherapy may be considered when systemic dexamethasone is decreased to $\leq 4$ $\mathrm{mg}$ /day and enhancing tumor burden is classified as SD, PR, or CR on follow-up scan, or when relevant treatment-related toxicity has resolved to grade $\leq 1$ or pre-treatment baseline.

\section{Immunotherapy Response Assessment in Neuro-Oncology (iRANO) Criteria}

The immunotherapy response assessment in neuro-oncology (iRANO) guidelines incorporate criteria previously defined by the RANO working committee to define CR, PR, 
minor response, SD, PD, and non-evaluable, for patients with malignant glioma, ${ }^{16}$ lowgrade glioma ${ }^{40}$ and brain metastases. ${ }^{18}$ The key component of iRANO is specific additional guidance for the determination of PD among neuro-oncology patients undergoing immunotherapy (Table 1; Figure 2). Specifically, iRANO advocates the confirmation of radiographic progression in appropriate patients defined by clinical status and time from initiation of immunotherapy.

Among patients who demonstrate imaging findings that meet RANO criteria for $\mathrm{PD}^{16-18}$ within six months of initiating immunotherapy including the development of new lesions, it may be appropriate to consider obtaining confirmation of radiographic progression on follow-up imaging before defining the patient a treatment failure provided that the patient is not experiencing new or worsened significant neurologic deficits. Such patients may be allowed a window of three months prior to confirming progression using the scan which first revealed initial progressive changes as the new reference scan for comparison with subsequent imaging studies. If RANO criteria for PD are met on the follow up scan at three months later, treatment failure should be assumed, and the date of PD should be back-dated to the initial date when it was identified (Table 1). Patients who develop significant new or worsened neurologic deficits not due to co-morbid event or change in co-administered medication at any time within the three months follow-up window should be designated as a treatment failure and should discontinue immunotherapy. For these patients, the date of actual tumor progression should also be back-dated to the date radiographic PD was initially identified.

If radiographic findings at the three month follow-up meet criteria for $\mathrm{SD}, \mathrm{PR}$, or $\mathrm{CR}$ according to RANO criteria ${ }^{16-18}$ compared to the original scan meeting criteria for progression, and there are no new or worsened neurologic deficits, such patients should be considered as deriving clinical benefit from therapy and allowed to continue treatment.

Patients who develop worsening radiographic findings compared to the pre-treatment baseline scan more than six months from immunotherapy initiation are expected to have a low likelihood of ultimately deriving clinical benefit and should be considered a treatment failure with a recommendation to discontinue their current therapy.

In summary, we have integrated guidance from the irRC regarding interpretation of progressive imaging findings with existing RANO criteria to form the iRANO guidelines. Comparison of the key features associated with RANO, irRC and iRANO are summarized in Table 2.

Although application of immunotherapies for neuro-oncology patients is in early stages and much remains to be learned, iRANO provides guidelines that can be applied to provide consistent metrics in clinical trials as well daily practice. In particular, these guidelines shall raise awareness of the possibility of potentially misleading early progressive radiographic changes following initiation of immunotherapy, as well as guidance for responding to these changes in order to decrease the likelihood of inappropriate premature therapy discontinuation. It is the expectation of the multinational, multidisciplinary co-authorship of this manuscript that the iRANO guidelines put forth herein will be amended successively to 
improve their utility as further experience and systematic data from ongoing immunotherapy trials in neuro-oncology accumulate.

\section{Corticosteroids}

Patients with brain tumors frequently develop peritumoral edema requiring treatment with corticosteroids. Dexamethasone is the most commonly used corticosteroid. ${ }^{41,42}$ In addition to systemic side effects, dexamethasone can have profound effects on contrast enhancement for neuroimaging studies and on the immune system, especially T cells. ${ }^{43}$ In preclinical studies, administration of dexamethasone to rats bearing intracranial C6 glioblastomas dosedependently decreased intratumoral infiltration by lymphocytes and microglial cells ${ }^{44}$, and limited cytokine-mediated antitumor effects and survival of rats bearing 9L gliomas. ${ }^{45}$

Several clinical studies have demonstrated that dexamethasone can inhibit maturation of dendritic cells and consequently their potential for antigen presentation. ${ }^{46,47}$ In cancer patients receiving immunotherapy, dexamethasone can also impair natural killer (NK) cell activity. ${ }^{48}$ In glioblastoma patients, treatment with dexamethasone favors the emergence of a population of $\mathrm{CD} 14^{+}$HLA.DR ${ }^{\text {low/neg }}$ monocytes that inhibit $\mathrm{T}$ cell proliferation. ${ }^{49}$

Most of the data on the impact of corticosteroids on immune system activity derive from evaluation of relatively high dosing schedules. In contrast, minimal data exists on the effects of differential doses ${ }^{50,51}$ while the long-term effects of low/moderate dexamethasone doses on immune cell function remain unclear. Nonetheless, given its potential negative effects on dendritic cell, T-cell and NK cell function, dexamethasone doses and duration of therapy should be limited to the minimum amount required to control neurological symptoms.

As a general guideline, patients enrolling in immunotherapy trials should be as little dexamethasone as possible prior to treatment initiation. During the course of treatment, if pseudoprogression occurs, higher doses of corticosteroids may be necessary to control symptoms. While this may potentially reduce immunotherapy efficacy, available data at present are inconclusive. In a trial of ipilimumab for brain metastases from melanoma, patients who required corticosteroids during study therapy had a worse outcome. ${ }^{52}$ Although this could be due to a negative effect of corticosteroids on immune function, it is also possible that the group requiring corticosteroids had larger tumors and worse prognostic factors.

Of note, patients who require increased corticosteroids within two weeks of MRI assessment relative to the dose taken at the time of the prior assessment, cannot be classified as CR, PR, or SD and should be classified as non-evaluable at that time point. Conversely, patients who decrease corticosteroids within two weeks of MRI assessment, relative to the dose taken at the time of the prior assessment, cannot be classified as PD and should be classified as nonevaluable.

\section{Conclusion}

We propose updated response assessment criteria for the evaluation of neuro-oncology patients undergoing immunotherapy. These recommendations integrate the framework of 
response assessment established by the RANO working group for malignant glioma, ${ }^{16}$ lowgrade glioma ${ }^{40}$, and brain metastases ${ }^{18}$ with guidance for confirmation of progression as originally advocated by the irRC criteria to guide clinical decision making. The iRANO guidelines specifically address interpretation of initial progressive imaging findings in the context of neuro-oncology patients with a goal of decreasing the likelihood of premature discontinuation of potentially beneficial therapies while ensuring maximum patient safety. The iRANO guidelines will inevitably require future amendment including possible incorporation of advanced imaging techniques, once sufficient experience and expertise are acquired for each of the major classes of immune-based therapies among neuro-oncology patients. Prospective evaluation of the iRANO criteria in brain tumor immunotherapy trials for neuro-oncology patients will be required to confirm their ultimate clinical utility.

\section{Search strategy and selection criteria}

A systematic search of PubMed was undertaken between January, 1980, and March, 2015, with (combinations of) the key words: "glioma", "glioblastoma", "immunotherapy", "imaging", "corticosteroid", and "response criteria". Articles were also identified through searches of the authors' own files. Only papers published in English were considered. The final reference list was generated on the basis of originality and relevance to the broad scope of this Review.

\section{Supplementary Material}

Refer to Web version on PubMed Central for supplementary material.

\section{Acknowledgment}

The authors thank the following iRANO working group members for their critical review on the manuscript: PierreYves Dietrich, MD (University of Geneva), Gavin Dunn, MD, PhD (Washington University), Gene Hwang, MD (Children National Washington DC), Michael Lim, MD (Johns Hopkins University), Pedro Lowenstein, MD, PhD (University of Michigan), James M. Markert, MD (University of Alabama at Birmingham), Christopher Moertel MD (University of Minnesota), Solmaz Sahebjam, MD (University of South Florida, Moffitt Cancer Center), Dewen Yang, MD, PhD (ICON Medical Imaging, Inc.). This work was supported by funding from NIH/NCI (R21 CA177787) to HO.

HO received royalties for licensing of his inventions from Stemline Therapeutics and Intrexon. MW received personal fees from Celldex, Immunocelular, Northwest Biotherapeutics and Magforce, grants from Acceleron, Actelion, Alpinia Institute, Bayer and Piqur, grants and personal fees from Isarna, MSD, Merck (EMD), Roche and Novocure. MRG received personal fees from Merck, Genentech, Abbvie, Wellcome Trust, Cell Medica and EMD Serono. WW received personal fees from Celldex and Prime Oncology, grants from MSD, Boehringer Ingelheim and Apogenix, grants and personal fees from Roche. BME received grants from Hoffman La Roche/Genentech and Siemens Healthcare. AAB received non-financial supports from Merck Serono and Pfizer. EF received nonfinancial support from Hoffman-La Roche. LN received advisory board fees from Amgen. WBP received personal fees from Celldex and Tocagen. RP received grants from Northwest Biotherapeutics. PYW received speaker's bureau fees from Merck, advisory board fees from AbbVie, Cavion, Celldex, Cubist, Genentech/Roche, Midatech, Momenta, Novartis, Novocure, SigmaTau and Vascular Biogenics. DAR received speaker's bureau fees from Roche/Genentech and Merck, advisory board fees from Novartis and Stemline Therapeutics, research grants from Celldex Therapeutics and Incyte.

MRG is an NIH employee. The following authors are funded by the NIH: HO, BME, IFP, AP, WBP, RP, JHS, PYW and DAR. 


\section{REFERENCES}

1. Kantoff PW, Higano CS, Shore ND, et al. Sipuleucel-T immunotherapy for castration-resistant prostate cancer. N Engl J Med. 2010; 363(5):411-22. [PubMed: 20818862]

2. Hodi FS, O'Day SJ, McDermott DF, et al. Improved survival with ipilimumab in patients with metastatic melanoma. N Engl J Med. 2010; 363(8):711-23. [PubMed: 20525992]

3. Robert C, Long GV, Brady B, et al. Nivolumab in previously untreated melanoma without BRAF mutation. N Engl J Med. 2015; 372(4):320-30. [PubMed: 25399552]

4. Robert C, Ribas A, Wolchok JD, et al. Anti-programmed-death-receptor-1 treatment with pembrolizumab in ipilimumab-refractory advanced melanoma: a randomised dose-comparison cohort of a phase 1 trial. Lancet. 2014; 384(9948):1109-17. [PubMed: 25034862]

5. Sundar R, Cho BC, Brahmer JR, Soo RA. Nivolumab in NSCLC: latest evidence and clinical potential. Therapeutic advances in medical oncology. 2015; 7(2):85-96. [PubMed: 25755681]

6. Maude SL, Frey N, Shaw PA, et al. Chimeric antigen receptor T cells for sustained remissions in leukemia. N Engl J Med. 2014; 371(16):1507-17. [PubMed: 25317870]

7. Grupp SA, Kalos M, Barrett D, et al. Chimeric antigen receptor-modified T cells for acute lymphoid leukemia. N Engl J Med. 2013; 368(16):1509-18. [PubMed: 23527958]

8. Reardon DA, Freeman G, Wu C, et al. Immunotherapy advances for glioblastoma. Neuro-oncology. 2014; 16(11):1441-58. [PubMed: 25190673]

9. Wainwright DA, Nigam P, Thaci B, Dey M, Lesniak MS. Recent developments on immunotherapy for brain cancer. Expert opinion on emerging drugs. 2012; 17(2):181-202. [PubMed: 22533851]

10. Jackson CM, Lim M, Drake CG. Immunotherapy for brain cancer: recent progress and future promise. Clinical cancer research: an official journal of the American Association for Cancer Research. 2014; 20(14):3651-9. [PubMed: 24771646]

11. Miller AB, Hoogstraten B, Staquet M, Winkler A. Reporting results of cancer treatment. Cancer. 1981; 47(1):207-14. [PubMed: 7459811]

12. Therasse P, Arbuck SG, Eisenhauer EA, et al. New guidelines to evaluate the response to treatment in solid tumors. European Organization for Research and Treatment of Cancer, National Cancer Institute of the United States, National Cancer Institute of Canada. J Natl Cancer Inst. 2000; 92(3): 205-16. [PubMed: 10655437]

13. Macdonald DR, Cascino TL, Schold SC Jr. Cairncross JG. Response criteria for phase II studies of supratentorial malignant glioma. Journal of Clinical Oncology. 1990; 8(7):1277-80. [PubMed: 2358840]

14. Brandsma D, Stalpers L, Taal W, Sminia P, van den Bent MJ. Clinical features, mechanisms, and management of pseudoprogression in malignant gliomas. The lancet oncology. 2008; 9(5):453-61. [PubMed: 18452856]

15. Chinot OL, Macdonald DR, Abrey LE, Zahlmann G, Kerloeguen Y, Cloughesy TF. Response assessment criteria for glioblastoma: practical adaptation and implementation in clinical trials of antiangiogenic therapy. Current neurology and neuroscience reports. 2013; 13(5):347. [PubMed: 23529375]

16. Wen PY, Macdonald DR, Reardon DA, et al. Updated response assessment criteria for high-grade gliomas: response assessment in neuro-oncology working group. J Clin Oncol. 2010; 28(11): 1963-72. [PubMed: 20231676]

17. van den Bent M, Wefel J, Schiff D, et al. Response assessment in neuro-oncology (a report of the RANO group): assessment of outcome in trials of diffuse low-grade gliomas. The lancet oncology. 2011; 12(6):583-93. [PubMed: 21474379]

18. Lin NU, Lee EQ, Aoyama H, et al. Proposed Response Assessment Criteria for Brain Metastases: Response Assessment in Neuro-Oncology (RANO) Working Group. The lancet oncology. 2015 In Press.

19. Radbruch A, Fladt J, Kickingereder P, et al. Pseudoprogression in patients with glioblastoma: clinical relevance despite low incidence. Neuro-oncology. 2014; 17(1):151-9. [PubMed: 25038253] 
20. de Wit MC, de Bruin HG, Eijkenboom W, Sillevis Smitt PA, van den Bent MJ. Immediate postradiotherapy changes in malignant glioma can mimic tumor progression. Neurology. 2004; 63(3): 535-7. [PubMed: 15304589]

21. Brandes AA, Franceschi E, Tosoni A, et al. MGMT promoter methylation status can predict the incidence and outcome of pseudoprogression after concomitant radiochemotherapy in newly diagnosed glioblastoma patients. J Clin Oncol. 2008; 26(13):2192-7. [PubMed: 18445844]

22. Wolchok JD, Hoos A, O'Day S, et al. Guidelines for the evaluation of immune therapy activity in solid tumors: immune-related response criteria. Clinical cancer research: an official journal of the American Association for Cancer Research. 2009; 15(23):7412-20. [PubMed: 19934295]

23. Hoos A. Evolution of end points for cancer immunotherapy trials. Annals of oncology: official journal of the European Society for Medical Oncology/ESMO. 2012; 23(Suppl 8):viii47-52. [PubMed: 22918928]

24. Hoos A, Eggermont AM, Janetzki S, et al. Improved endpoints for cancer immunotherapy trials. J Natl Cancer Inst. 2010; 102(18):1388-97. [PubMed: 20826737]

25. Okada H, Kalinski P, Ueda R, et al. Induction of CD8+ T-cell responses against novel gliomaassociated antigen peptides and clinical activity by vaccinations with a-type 1 polarized dendritic cells and polyinosinic-polycytidylic acid stabilized by lysine and carboxymethylcellulose in patients with recurrent malignant glioma. Journal of clinical oncology: official journal of the American Society of Clinical Oncology. 2011; 29(3):330-6. [PubMed: 21149657]

26. Topalian SL, Hodi FS, Brahmer JR, et al. Safety, activity, and immune correlates of anti-PD-1 antibody in cancer. N Engl J Med. 2012; 366(26):2443-54. [PubMed: 22658127]

27. Topalian SL, Sznol M, McDermott DF, et al. Survival, durable tumor remission, and long-term safety in patients with advanced melanoma receiving nivolumab. J Clin Oncol. 2014; 32(10): 1020-30. [PubMed: 24590637]

28. Hamid O, Robert C, Daud A, et al. Safety and tumor responses with lambrolizumab (anti-PD-1) in melanoma. N Engl J Med. 2013; 369(2):134-44. [PubMed: 23724846]

29. Okada H, Pollack IF. Do we need novel radiologic response criteria for brain tumor immunotherapy? Expert Rev Neurother. 2011; 11(5):619-22. [PubMed: 21539483]

30. Wolchok JD, Kluger H, Callahan MK, et al. Nivolumab plus ipilimumab in advanced melanoma. N Engl J Med. 2013; 369(2):122-33. [PubMed: 23724867]

31. Brahmer JR, Tykodi SS, Chow LQ, et al. Safety and activity of anti-PD-L1 antibody in patients with advanced cancer. N Engl J Med. 2012; 366(26):2455-65. [PubMed: 22658128]

32. Hodi FS, Lawrence D, Lezcano C, et al. Bevacizumab plus Ipilimumab in Patients with Metastatic Melanoma. Cancer immunology research. 2014; 2(7):632-42. [PubMed: 24838938]

33. Okada H, Kohanbash G, Zhu X, et al. Immunotherapeutic approaches for glioma. Critical reviews in immunology. 2009; 29(1):1-42. [PubMed: 19348609]

34. Kirkwood JM, Lorigan P, Hersey P, et al. Phase II trial of tremelimumab (CP-675,206) in patients with advanced refractory or relapsed melanoma. Clinical cancer research: an official journal of the American Association for Cancer Research. 2010; 16(3):1042-8. [PubMed: 20086001]

35. Huang RY, Neagu MR, Reardon DA, Wen PY. Pitfalls in the neuroimaging of glioblastoma in the era of antiangiogenic and immuno/targeted therapy - detecting illusive disease, defining response. Frontiers in neurology. 2015; 6:33. [PubMed: 25755649]

36. Sampson JH, Heimberger AB, Archer GE, et al. Immunologic escape after prolonged progressionfree survival with epidermal growth factor receptor variant III peptide vaccination in patients with newly diagnosed glioblastoma. Journal of clinical oncology: official journal of the American Society of Clinical Oncology. 2010; 28(31):4722-9. [PubMed: 20921459]

37. Hoos A, Parmiani G, Hege K, et al. A clinical development paradigm for cancer vaccines and related biologics. Journal of immunotherapy. 2007; 30(1):1-15. [PubMed: 17198079]

38. Pollack IF, Jakacki RI, Butterfield LH, et al. Antigen-specific immune responses and clinical outcome after vaccination with glioma-associated antigen peptides and polyinosinic-polycytidylic acid stabilized by lysine and carboxymethylcellulose in children with newly diagnosed malignant brainstem and nonbrainstem gliomas. J Clin Oncol. 2014; 32(19):2050-8. [PubMed: 24888813] 
39. Brandes AA, Tosoni A, Spagnolli F, et al. Disease progression or pseudoprogression after concomitant radiochemotherapy treatment: pitfalls in neurooncology. Neuro-oncology. 2008; 10(3):361-7. [PubMed: 18401015]

40. van den Bent MJ, Wefel JS, Schiff D, et al. Response assessment in neuro-oncology (a report of the RANO group): assessment of outcome in trials of diffuse low-grade gliomas. The lancet oncology. 2011; 12(6):583-93. [PubMed: 21474379]

41. Kaal EC, Vecht CJ. The management of brain edema in brain tumors. Current opinion in oncology. 2004; 16(6):593-600. [PubMed: 15627023]

42. Dietrich J, Rao K, Pastorino S, Kesari S. Corticosteroids in brain cancer patients: benefits and pitfalls. Expert review of clinical pharmacology. 2011; 4(2):233-42. [PubMed: 21666852]

43. Ashwell JD, Vacchio MS, Galon J. Do glucocorticoids participate in thymocyte development? Immunology today. 2000; 21(12):644-6. [PubMed: 11188796]

44. Badie B, Schartner JM, Paul J, Bartley BA, Vorpahl J, Preston JK. Dexamethasone-induced abolition of the inflammatory response in an experimental glioma model: a flow cytometry study. Journal of neurosurgery. 2000; 93(4):634-9. [PubMed: 11014542]

45. Benedetti S, Pirola B, Poliani PL, et al. Dexamethasone inhibits the anti-tumor effect of interleukin 4 on rat experimental gliomas. Gene therapy. 2003; 10(2):188-92. [PubMed: 12571648]

46. Piemonti L, Monti P, Allavena $\mathrm{P}$, et al. Glucocorticoids affect human dendritic cell differentiation and maturation. Journal of immunology. 1999; 162(11):6473-81.

47. Matasic R, Dietz AB, Vuk-Pavlovic S. Dexamethasone inhibits dendritic cell maturation by redirecting differentiation of a subset of cells. Journal of leukocyte biology. 1999; 66(6):909-14. [PubMed: 10614771]

48. Hsu AK, Quach H, Tai T, et al. The immunostimulatory effect of lenalidomide on NK-cell function is profoundly inhibited by concurrent dexamethasone therapy. Blood. 2011; 117(5):160513. [PubMed: 20978269]

49. Gustafson MP, Lin Y, New KC, et al. Systemic immune suppression in glioblastoma: the interplay between CD14+HLA-DRlo/neg monocytes, tumor factors, and dexamethasone. Neuro-oncology. 2010; 12(7):631-44. [PubMed: 20179016]

50. Vecht CJ, Haaxma-Reiche H, Noordijk EM, et al. Treatment of single brain metastasis: radiotherapy alone or combined with neurosurgery? Annals of Neurology. 1993; 33(6):583-90. [PubMed: 8498838]

51. Wolfson AH, Snodgrass SM, Schwade JG, et al. The role of steroids in the management of metastatic carcinoma to the brain. A pilot prospective trial. American journal of clinical oncology. 1994; 17(3):234-8. [PubMed: 8192109]

52. Margolin K, Ernstoff MS, Hamid O, et al. Ipilimumab in patients with melanoma and brain metastases: an open-label, phase 2 trial. The lancet oncology. 2012; 13(5):459-65. [PubMed: 22456429] 
(A)

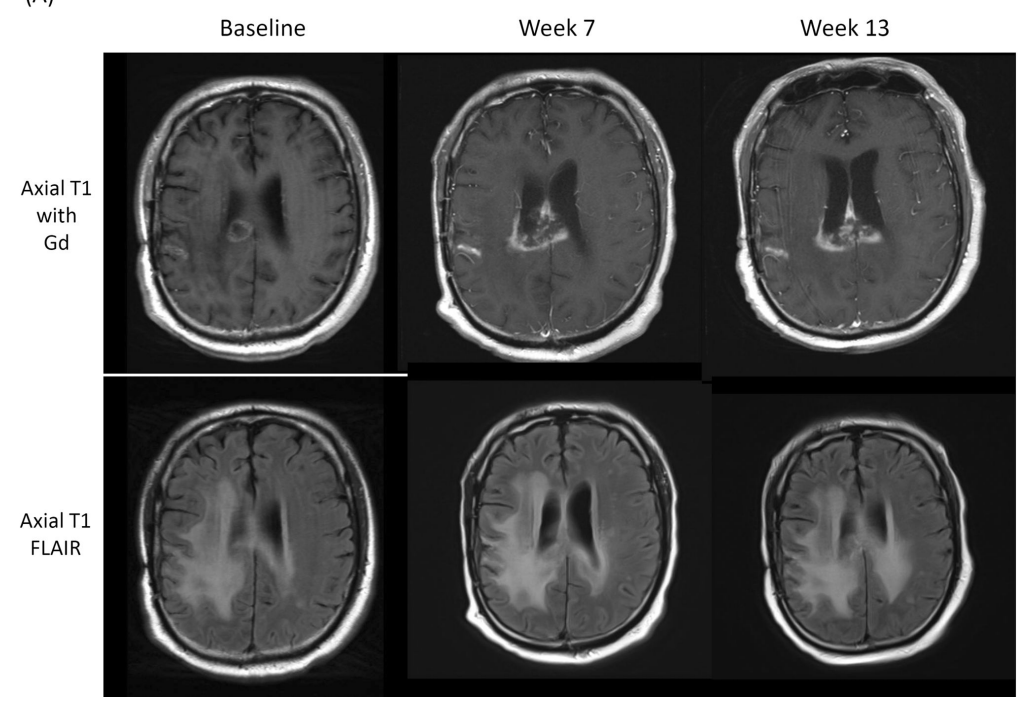

(B)

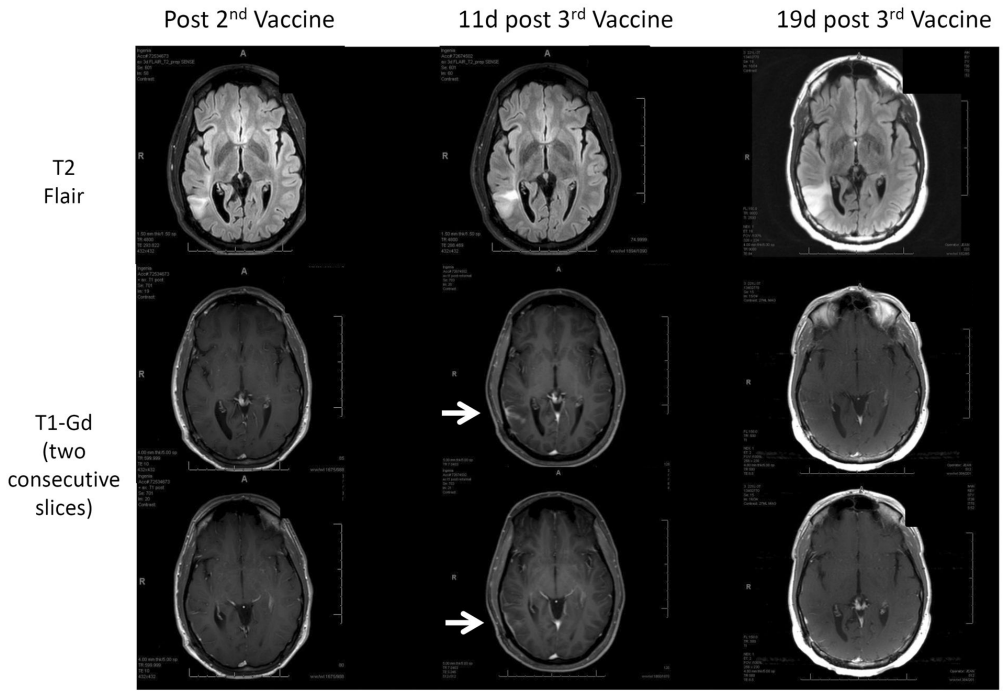

Figure 1.

(A) Axial T1 contrast Gd-enhanced and FLAIR images obtained prior to, and 7 and 13 weeks after initiation of CTLA-4 immune checkpoint blockade. ${ }^{35}$ (B) Axial T2 FLAIR and $\mathrm{T} 1 \mathrm{Gd}$-enhanced images obtained post $2^{\text {nd }}$ (left), at 11 days post $3^{\text {rd }}$ (middle) and 19 days post $3^{\text {rd }}$ (right) vaccinations (NCT01678352) in a patient with recurrent WHO grade II oliodendroglioma. 


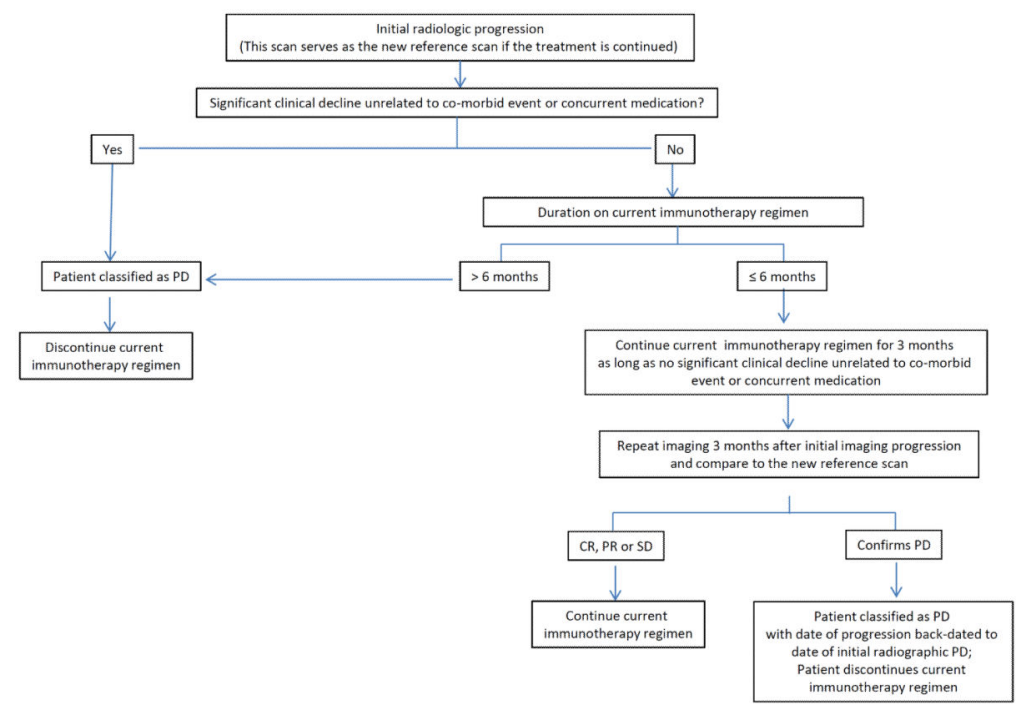

Figure 2.

Algorithm for evaluation of progressive imaging findings among neuro-oncology patients undergoing immune-based therapies. 


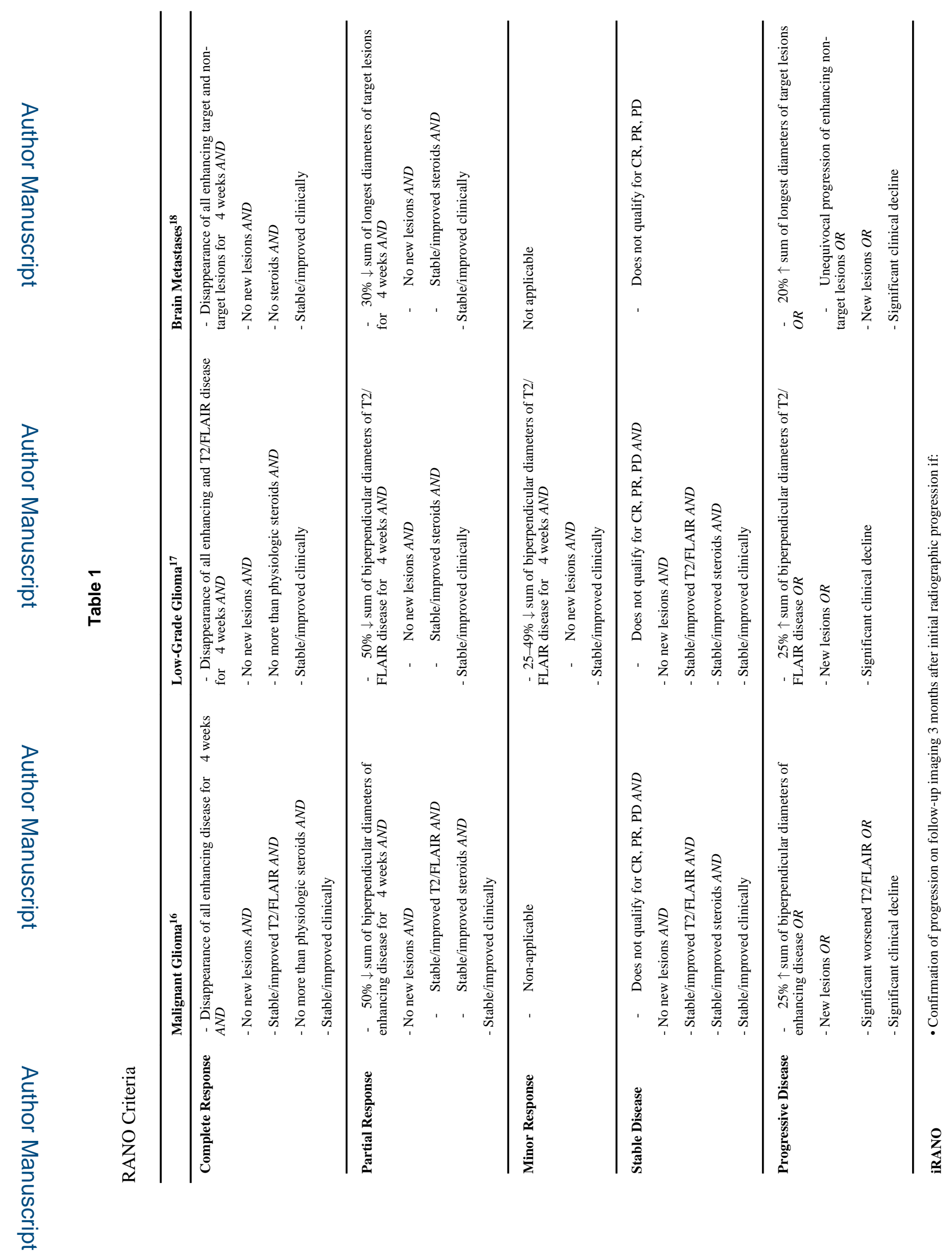

Lancet Oncol. Author manuscript; available in PMC 2016 November 01. 


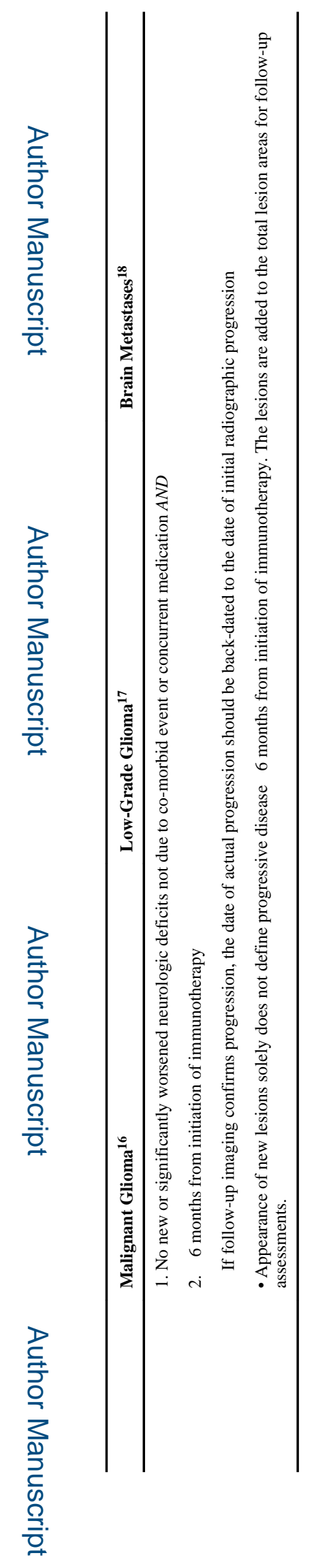


Table 2

Key Considerations: RANO, irRC and iRANO

\begin{tabular}{|l|l|l|l|l|}
\hline & RANO & irRC & $\begin{array}{l}\text { iRANO (if } \leq 6 \text { months } \\
\text { after start of } \\
\text { immunotherapy) }\end{array}$ & $\begin{array}{l}\text { iRANO (if }>\text { 6 months } \\
\text { after start of } \\
\text { immunotherapy) }\end{array}$ \\
\hline $\begin{array}{l}\text { Is a repeat scan required to confirm radiographic } \\
\text { PD for patients without significant clinical } \\
\text { decline? }\end{array}$ & No & Yes & Yes & No \\
\hline $\begin{array}{l}\text { Minimal time interval for confirmation of } \\
\text { progression for patients without significant } \\
\text { clinical decline? }\end{array}$ & Not applicable & $\geq 4$ weeks & $\geq 3$ months & Not applicable \\
\hline $\begin{array}{l}\text { Is further immunotherapy treatment allowed after } \\
\text { initial radiographic PD (if clinically stable) } \\
\text { pending progression confirmation }\end{array}$ & Not applicable & Yes & Yes & Not applicable \\
\hline Does a new lesion define PD? & Yes & No & No & Yes \\
\hline
\end{tabular}

